\title{
Receptor-Independent Metabolic Effects of Thiazolidinediones in Astrocytes
}

\author{
Candan Akar, Sergey Kalinin, Vitaliy Gavrilyuk, Alessandra Spagnolo, Guy Weinberg, and Douglas \\ L Feinstein
}

Department of Anesthesiology, University of Illinois, Chicago, IL, USA

\begin{abstract}
Thiazodinedione (TZD) agonists of the peroxisome proliferator activated receptor gamma (PPAR $\gamma$ ) exert metabolic effects in glial cells. In primary astrocytes, TZDs are cytoprotective and have anti-inflammatory actions; in contrast, in glioma cells TZDs are cytotoxic. Although PPAR $\gamma$ is considered their primary target, TZDs including pioglitazone and troglitazone also bind to a mitochondrial protein MitoNEET; whether their metabolic effects are mediated by activation of PPAR $\gamma$ or MitoNEET are not known. We generated PPAR $\gamma$ null astrocytes by crossing a PPAR $\gamma$ floxxed mouse with a transgenic line expressing CRE recombinase under control of the GFAP promoter. PPAR $\gamma$ deficient astrocytes showed reduced lactate production under basal conditions and in response to pioglitazone; however at later times similar levels of lactate were produced. In the presence of troglitazone lactate production was similar in PPAR $\gamma$ null cells as wildtype astrocytes. In astrocytes in which MitoNEET expression was reduced using siRNA, basal lactate production was lower than control cells, however the cells increased lactate production in response to TZDs. When MitoNEET was decreased in the PPAR $\gamma$ null astrocytes, responses to TZDs were reduced compared to non-infected cells. These results indicate that metabolic effects of TZDs are not exclusively mediated via PPAR $\gamma$, but involve binding to MitoNEET. Real time PCR revealed significantly greater MitoNEET mRNA in glioma cells than astrocytes. Differences in MitoNEET expression or activity could therefore contribute to differential effects of TZDs on astrocyte versus glioma cells.
\end{abstract}

Keywords: Brain, PPAR, lactate, mitochondria, MitoNEET.

\section{INTRODUCTION}

Thiazolidinediones (TZDs) are synthetic compounds used as oral anti-diabetic drugs. TZDs, which include Troglitazone, Pioglitazone, and Rosiglitazone, are agonists of peroxisome proliferator activated receptor gamma (PPAR $\gamma$ ) of the PPAR family. PPAR $\gamma$ is the best characterized of the three major isoforms $(\alpha, \beta / \delta$, and $\gamma)$, in part due to its therapeutic potential for treatment of diabetes [1] and related consequences such as metabolic syndrome [2]. The glucose-lowering effect of TZDs has been generally assumed to be due to activation of PPAR $\gamma$ which is known to increase transcription of insulin-sensitive genes [3, 4]. In addition to effects on energy and fuel metabolism, several studies report TZD involvement in suppression of cell proliferation, induction of cytotoxicity; and perturbation of mitochondrial function. In these studies the rapid occurrence of agonist induced effects, the lack of correlation between the effects and the affinity for PPAR $\gamma$, and the incability of antagonists to block the effects suggest PPAR $\gamma$ independent action by TZDs.

In primary cultures of astrocytes, we reported that TZDs caused a rapid increase in glucose consumption and lactate production, associated with an rapid decrease in mitochondrial membrane potential followed by a subsequent hyperpolarization [5]. Similarly, in isolated rat liver, infusion of

*Address correspondence to this author at Department of Anesthesiology, University of Illinois; and Jesse Brown Veterans Affairs Hospital, Chicago, IL, USA; Tel: 312-355-1665; Fax: 312-996-9672; E-mail: dlfeins@uic.edu troglitazone increased lactate production in less than $10 \mathrm{~min}$ [6]. Rapid effects on mitochondria were reported with ciglitazone which increased ROS production [7] in astrocytes, and similar effects were observed using 10 to $20 \mathrm{uM}$ pioglitazone or troglitazone in mouse astrocytes and astrocytoma cells [8]. These findings suggest that TZDs may exert direct and rapid effects on mitochondrial respiration leading to changes in glucose metabolism and fuel substrate specificity. Differential effects of TZDs on mitochondrial respiration may account for the selective ability of TZDs to induce toxicity in transformed glial cells .

A possible site of TZD action on mitochondria may be the recently described mitochondrial protein "mitoNEET", [9]. mitoNEET was identified by saturable binding of labeled pioglitazone to crude mitochondrial membranes from bovine brain and several other tissues. The binding of pioglitazone to this protein was specific with half maximal binding occurring between 0.1 and $1 \mathrm{uM}$. After cross-linking with photoaffinity labeled pioglitazone a $17 \mathrm{kD}$ protein was determined to be the binding site. Consequent purification and proteomic analysis revealed a novel protein containing the sequence Asp-Glu-Glu-Tyr ( 'NEET'). mitoNEET was also found to be associated with several other mitochondrial proteins, including components of the pyruvate dehydrogenase complex, suggesting a means by which TZD binding to mitoNEET could block pyruvate driven respiration.

In this study we investigated the involvement of mitoNEET in mediation of PPAR $\gamma$ independent metabolic ef- 
fects of TZDs. Our data show that knockdown of MitoNEET in astrocytes accomplished by siRNA leads to an increase in lactate production; In contrast, the effects of pioglitazone on lactate production are comparable in PPAR $\gamma$ expressing versus null cells.

\section{EXPERIMENTAL PROCEDURES}

\section{Cells}

Primary astrocytes were prepared from cerebral cortices of postnatal day 1 C57BL/6 or PPAR $\gamma$ null mice as described previously [10]. Mouse GL261 glioma cells were grown as previously described [8]. The cells were grown in DMEM containing $25 \mathrm{mM}$ glucose, $10 \%$ FCS and antibiotics (penicillin and streptomycin) by changing the medium every three days for two weeks before using for experiments.

\section{$\operatorname{PPAR} \gamma$ knockout}

PPAR $\gamma$ conditional astrocyte knockout mice were created as previously described by crossing the PPAR $\gamma$ floxxed mice (PPAR f/f, Dr.F. Gonzalez, NIH) with mice having GFAP-driven Cre expression (Cre +/-, Dr. A. Messing, U. Madison). The desired F2 generation mice PPAR $\gamma$ f/f : Cre +/-) were crossed to parental PPAR $\gamma$ f/f : Cre -/- mice to generate $50 \%$ knockout (KO) and $50 \%$ wild type (WT) littermates

\section{Infection of Cells with siRNA and Drug Treatment}

Adenovirus particles containing siRNA for mitoNEET were designed and produced from Galapagos Genomics (Mechelen, Belguim). The RNA duplex targeted the 3'end of the mitoNEET mRNA sequence bases 415 5'-AAACCTAAT GGACAGTTGCGA.-3' 435 which spans the stop codon at base 422 . The particles were added to DMEM containing $1 \%$ FBS and penicillin/streptomycin. $500 \mu \mathrm{l} /$ well of this medium were added to astrocytes on 6 well plates to give a final concentration of $10 \mathrm{MOI} /$ cell. Control plates were treated with the same medium but without adenovirus. Plates were left in the incubator for $2 \mathrm{hrs}$ then the medium was changed to DMEM containing $10 \%$ FBS and penicillin/strepto-mycin. Total cellular protein levels were not significantly altered by infection. After 72 hours of incubation the cells were washed twice with low glucose $(5.6 \mathrm{mM})$ DMEM containing 1\% FBS. Cells were then treated with pioglitazone or troglitazone $(20 \mu \mathrm{M}$ in DMSO) or DMSO $(0.1 \%)$ in the same medium and incubated for $6 \mathrm{hrs}$. Samples were taken from the culture medium at 0 time point then every $2 \mathrm{hrs}$ for assessment of lactate levels. After $6 \mathrm{hrs}$ the medium was removed and $1 \mathrm{ml}$ Trizol reagent (Invitrogen, Carlsbad, CA) / well was added onto the cells. Trizol samples were frozen and kept at $-80^{\circ} \mathrm{C}$.

\section{Lactate Assay}

The lactate amounts in samples taken from the culture medium were determined enzymatically using assay kits following manufacturer's protocol with some modifications. Briefly, 5 ul of sample was incubated with $95 \mathrm{ul}$ of reagent (Trinity Biotech, Bray, Ireland) for $20 \mathrm{~min}$ at room temperature and then the absorbance was measured at $540 \mathrm{~nm}$. Lac- tate amounts were calculated by interpolation from a standard curves of L-lactate in $\mathrm{H}_{2} \mathrm{O}$.

\section{Determination of mitoNEET Knock Down}

Total RNA was isolated from Trizol samples by chloroform extraction and ethanol precipitation. $1 \mu \mathrm{g}$ of the RNA was converted into cDNA using random hexamer primers, and mRNA levels were determined by quantitative real time-PCR. The reactions were carried out in the presence of SYBRGreen diluted 1:10000 from stock solution (Molecular Probes, Eugene, OR) in Corbett Rotor-Gene real time PCR unit (Corbett Research, Sydney, Australia). The primers used for mouse mitoNEET (Cisd1, accession number NM 134007) were 5'-AAC CTA ATG GAC AGT TGC GAG GCT-3' forward and 5'-AAG GCC GAT GCC ATG GAT ATG AGA-3' reverse, which gave a 158 bp product. Relative mRNA concentrations were calculated from the take off point $\left(C_{t}\right)$ of reactions using manufacturer's software.

\section{Quantitative PCR}

Real time PCR was used to measure mitoNEET mRNA levels using forward primer 5'-CAA AGC TAT GGT GAA TCT TCAG and reverse primer 5'- GTG CCA TTC TAC GTA AAT CAG which generates a 158 bp product. Values were normalized to levels measured for beta-actin mRNA in the samples using primers 5' CCT GAA GTA CCC CAT TGA ACA and reverse 5'-CAC ACG CAG CTC ATT GTA GAA. PCR conditions were 35 cycles of denaturation at $94^{\circ} \mathrm{C}$ for $10 \mathrm{~s}$; annealing at $64^{\circ} \mathrm{C}$ for $15 \mathrm{~s}$; and extension at $72^{\circ} \mathrm{C}$ for $20 \mathrm{~s}$ on a Corbett Rotorgene Real-Time PCR unit (Corbett, Australia). PCR was done using Taq DNA polymerase (Invitrogen), and contained SYBR Green (SybrGreen110,000x concentrate, diluted 1:10,000; Molecular Probes, Eugene, OR). Relative mRNA concentrations were calculated from the takeoff point of reactions using manufacturer's software.

\section{Data Analysis}

Time dependent changes in lactate production between wildtype and PPAR $\gamma$ null cells was compared by 2-way repeated measures ANOVA, and considered significantly different if the time $\mathrm{x}$ cell type interaction effects $\mathrm{P}$ value was $<0.05$.

\section{RESULTS}

In order to determine whether the metabolic effects of pioglitazone on astrocyte metabolism are mediated through PPAR $\gamma$ we made use of PPAR $\gamma$ conditional astrocyte knockout mice [11]. In wildtype (WT) cells, incubation with pioglitazone or troglitazone induced significant time-dependent increases in lactate production as previously shown [5]. In PPAR $\gamma$ null cells, the baseline production of lactate was slightly, but significantly decreased compared to that in the WT cells (Fig. 1). Between 0 and $4 \mathrm{hr}$ the rate of production was reduced $28 \%$ compared to WT cells, however at $6 \mathrm{hr}$ levels were similar. In the presence of pioglitazone, lactate production was also increased in PPAR $\gamma$ null astrocytes although the absolute values were significantly lower than those in the WT cells between 0 and $4 \mathrm{hr}$, although again at $6 \mathrm{hr}$ levels were comparable. Because of lower production in vehicle treated cells, the magnitude of 


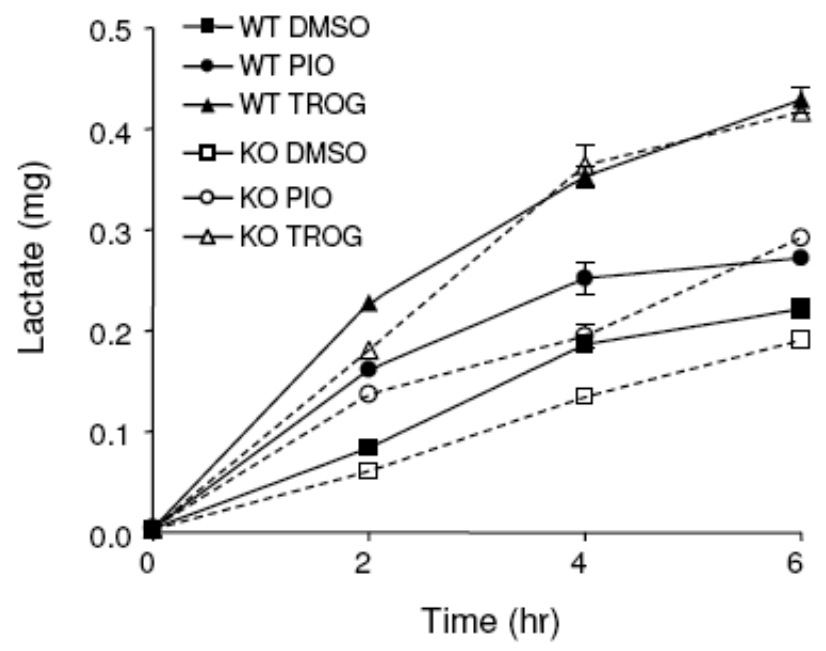

Fig. (1). Effect of TZDs on lactate production in PPAR $\gamma$ null astrocytes.

Astrocytes from PPAR $\gamma$ null (open symbols) and wild type (filled symbols) mice were incubated with $20 \mu \mathrm{M}$ pioglitazone (circles) or $20 \mu \mathrm{M}$ troglitazone (triangles in low glucose $(5.6 \mathrm{mM})$ DMEM containing 1\% FBS. Control (squares) cultures were incubated with medium plus the equivalent amount of vehicle (DMSO). Lactate levels in the culture media were determined at the indicated time points. The data is the mean \pm s.e.m. of $n=3$ measurements for each point. Two way ANOVA showed that lactate production was significantly different between wilt type and PPAR $\gamma$ null cells in the presence of vehicle $(\mathrm{P}=0.015)$ or pioglitazone $(\mathrm{P}=0.032)$; but was not different in the presence of troglitazone.

the increase due to pioglitazone versus control cells was similar in PPAR $\gamma$ null and WT cells (approximately $80 \%$ increase after $2 \mathrm{hr}$, and $40 \%$ increase at 4 and $6 \mathrm{hr}$ ). In con- trast, lactate production was comparably increased in the two cell types in the presence of troglitazone, which although having a lower affinity than pioglitazone for $\operatorname{PPAR} \gamma$, caused a greater increase in lactate production. These results suggest that metabolic effects of pioglitazone show a partial dependence on the presence of PPAR $\gamma$, but that this dependence is lost after longer times, or in the presence of a more potent metabolic induce such as troglitazone.

Since pioglitazone can also bind to the mitoNEET protein, we investigated a possible involvement of this protein in mediating the metabolic effects of TZDs (Fig. 2). Infection of primary mouse astocytes with 10 MOI adenovirus containing siRNA directed against mitoNEET reduced mitoNEET mRNA levels approximately $70 \%$ as compared to mock infected cells (Fig. 2A). The reduction in mitoNEET expression was associated with a slight but significant decrease in lactate production versus the non-infected cells (Fig. 2B); at 4 and $6 \mathrm{hr}$ levels were reduced about $30 \%$, compared to non-infected cells. However, treatment with pioglitazone significantly increased lactate production in the control cells as well as the siRNA treated cells; as did incubation with troglitazone. These results suggests that TZD can continue to influence astrocyte metabolism either via effects on residual MitoNEET, or through interactions with PPAR $\gamma$.

To determine the consequences of combined mitoNEET and PPAR $\gamma$ depletion, we compared the effects of infecting PPAR $\gamma$ null versus WT astrocytes with adenovirus containing siRNA (Fig. 3). In this study, basal production of lactate was similar in the mitoNEET depleted WT and PPAR $\gamma$ null cells; although a slight increase was seen after $6 \mathrm{hr}$ in null cells. In the presence of pioglitazone, lactate production increased over time in both cells types, however there was a significantly lower increase observed in the null cells com-
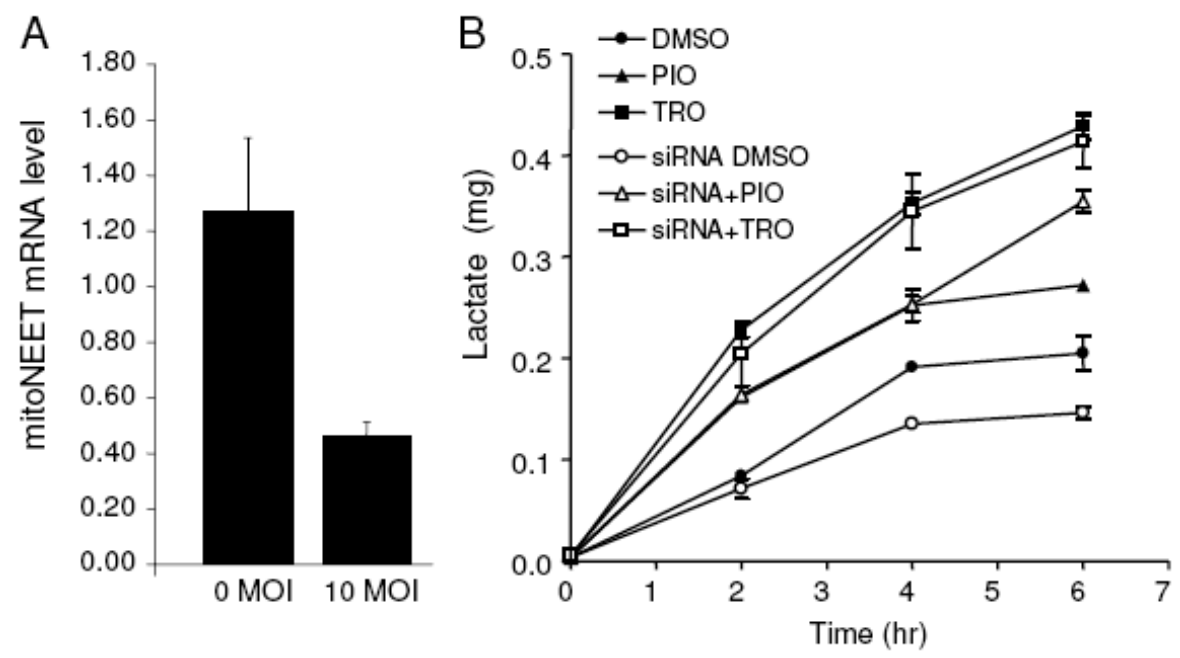

Fig. (2). Effect of mitoNEET depletion on lactate production in astrocytes.

Mouse astrocytes were incubated with adenovirus particles (10 MOI / cell), containing siRNA for mitoNEET (open symbols), in DMEM with $1 \%$ FBS or with medium alone (filled symbols) for two hours. The medium was then changed to DMEM with $10 \%$ FBS. After 72 hrs of incubation the cultures were treated with $20 \mu \mathrm{M}$ pioglitazone (circles) or troglitazone (triangles) in low glucose (5.6 mM) DMEM containing $1 \%$ FBS. Control (squares) cultures were incubated with medium plus the equivalent amount of vehicle. (A) After 6 hr incubation mitoNEET mRNA levels were determined by QPCR, normalized against $\beta$-tubulin mRNA levels. (B) Lactate levels were determined in the media at the indicated times. The data is the mean \pm s.e.m. of $n=3$ replicates. The production of lactate in the presence of vehicle was significantly different between control cells and mitoNEET depleted cells ( 2 way ANOVA, $\mathrm{P}=0.0035$ ). 


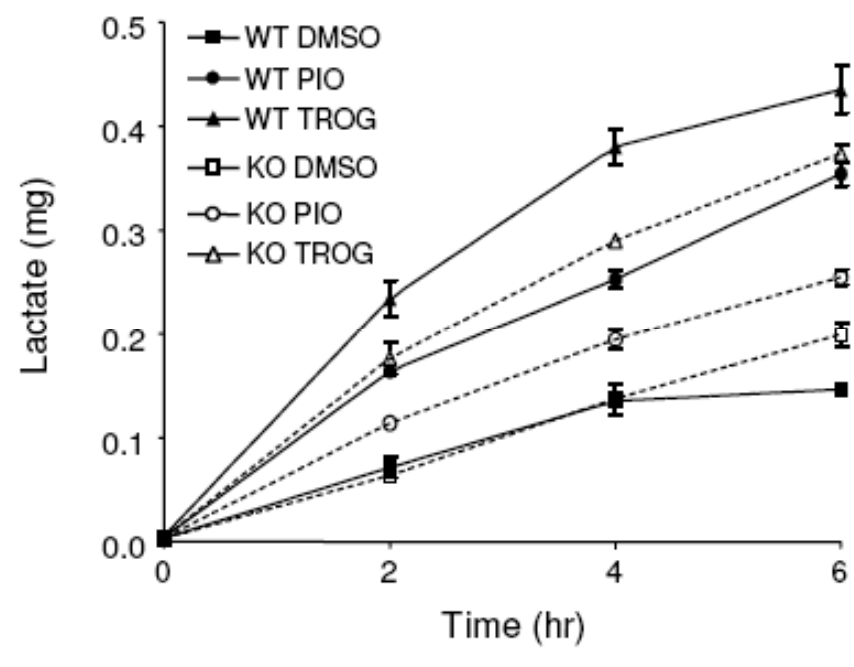

Fig. (3). Effect of mitoNEET depletion on lactate production in PPAR $\gamma$ null astrocytes.

Astrocyte cultures from PPAR $\gamma$ knockout (open symbols) and wild type (filled symbols) mice were incubated with mitoNEET siRNA containing adenovirus particles (10 MOI/cell), then after $72 \mathrm{hr}$ the media was changed and the cells were incubated in low glucose (5.6 mM) DMEM with $1 \%$ FCS and $20 \mu \mathrm{M}$ pioglitazone (circles) or $20 \mu \mathrm{M}$ troglitazone (triangles as in Fig. (3). Control (squares) cultures were incubated with medium plus the equivalent amount of vehicle. Lactate levels in the media were determined at the indicated time points. The data is the mean \pm s.e.m. of $n=3$ measurements. Lactate production was significantly different between the two cells types in the presence of pioglitazone ( 2 way ANOVA, $\mathrm{P}<$ $0.0001)$, but not in the presence of troglitazone $(\mathrm{P}=0.11)$.

pared to the WT cells (lactate levels increased at 6hr over $300 \%$ in the WT cells, but only about $40 \%$ in the PPAR $\gamma$ null cells). Likewise, the effect of troglitazone on lactate production was attenuated by mitoNEET depletion in the PPAR $\gamma$ null cells, but to a smaller and non-significantly different extent than that seen with pioglitazone.

Using quantitative real time PCR we measured relative mitoNEET mRNA levels in primary mouse astrocytes, and compared that to levels in mouse GL261 glioma cells (Fig. 4). Interestingly, mitoNEET levels were significantly higher (about 8-fold) in the glioma cells, possibly due to the higher metabolic state of the cells.

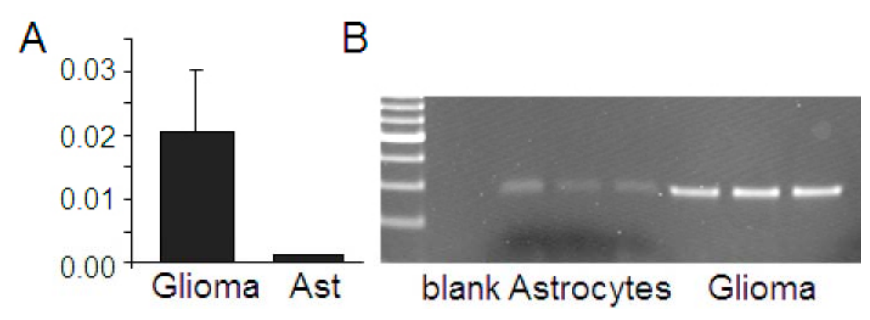

Fig. (4). MitoNEET mRNA levels in astrocytes versus glioma cells.

Total mRNA was isolated from primary mouse astrocytes and from mouse GL261 glioma cells, converted to cDNA, and relative levels of mitoNEET mRNA determined by quantitative real time PCR.

(A) The data is average of 3 measurements for each sample nor- malized to values for beta-actin mRNAs measured in the same samples. The gel shown in (B) confirms that the PCR products are the correct size generated from astrocytes and glioma cells.

\section{DISCUSSION}

In this report we have shown that the ability of two TZDs, pioglitazone and troglitazone to increase lactate production in astrocytes is not fully dependent on PPAR $\gamma$. Both basal lactate production as well as the increased production due to the presence of pioglitazone were significantly different between control astrocytes and astrocytes in which PPAR $\gamma$ was depleted; however by $6 \mathrm{hr}$ the lactate production was comparable in the two cell types. Furthermore, in the presence of troglitazone virtually identical rates of lactate production were observed. In contrast, depletion of an alternate target of TZDs, the mitoNEET protein present in mitochondria [9], led to a small decrease in the basal production of lactate; although the TZD-dependent increases were similar. Together these findings suggest that both PPAR $\gamma$ as well as mitoNEET contribute to the metabolic effects of TZDs in astrocytes; and this is supported by results showing that depletion of mitoNEET in the PPAR $\gamma$ null astrocytes led to a statistically significant reduction in pioglitazone-dependent lactate production (Fig. 3); and a lesser decrease in the presence of troglitazone.

Metabolic effects of TZDs have been reported for several cell types [12-17], and in many cases these effects occurred relatively rapidly or at TZD doses much higher than their binding affinity for PPAR $\gamma$, and in some studies, non TZD PPAR $\gamma$ ligands showed little or no metabolic effects. These results raised the possibility that these metabolic effects occurred in a PPAR $\gamma$ independent manner, which are consistent with the current findings in PPAR $\gamma$ null astrocytes.

Alternate sites of action for TZDs have been suggested $[18,19]$, and recently selective binding to the mitochondrial protein mitoNEET was demonstrated [9]. Using biochemical approaches to identify the binding site for pioglitazone, studies using photoaffinity labeled pioglitazone could only identify a single $17 \mathrm{kDa}$ protein that could be specifically crosslinked, which is expressed in the mitochondria; while binding studies using high specific activity radiolabeled pioglitazone Pio showed that MitoNEET was the only protein that could be labeled in whole cell lysates. MitoNEET is a member of a small family of proteins which contain a novel zing finger motif; but contains iron rather than zinc, and contains a $2 \mathrm{Fe}-2 \mathrm{~S}$ cluster that is released in a redox and pH dependent manner [20]. MitoNEET has been crystallized and a $1.5 \mathrm{~A}$ structure determined, which shows that pioglitazone stabilizes the homodimeric form of the protein against release of the $2 \mathrm{Fe}-2 \mathrm{~S}$ cluster $[21,22]$. The exact function(s) of mitoNeet remain to be clarified, but TZDs including pioglitazone induce a conformational change influencing overall mitochondria redox potential and respiration, and its association with subunits of PDH complex suggests it may be involved in regulation of pyruvate transport or metabolism. Mitochondria isolated from mitoNEET null mice show reduced oxidative capacity [23]. Our findings that when mitoNEET levels are decreased in PPAR $\gamma$ null cells the effect of pioglitazone also decreases suggests an involvement of mitoNEET in mediation of this effect. 
In summary, our results demonstrate that metabolic effects of TZDs in primary astrocytes are not fully dependent upon PPAR $\gamma$ activation, and implicate mitoNEET as an additional target for those actions. Initial analysis of mitoNEET expression in primary mouse astrocytes versus glioma cells suggests that mitoNEET is expressed at much higher levels in the tumor cells. This may in part account for the selective ability of TZDs to induce apoptosis in tumor cells [8, 24]. However, further studies where mitoNEET mRNA and protein levels can be reduced to varying degrees, or are completely abolished, are necessary to clarify the relative role of mitoNEET in mediation of TZD effects.

\section{ACKNOWLEDGEMENTS}

We than Jerry Colca (Metabolic Solutions Development) and Patrick Blanner (Pfizer) for providing advice and mitoNEET reagents.

\section{REFERENCES}

[1] Rangwala SM, Lazar MA. Peroxisome proliferator-activated receptor gamma in diabetes and metabolism. Trends Pharmacol Sci 2004; 25(6): 331-6.

[2] Pakala R, Kuchulakanti P, Rha SW, Cheneau E, Baffour R, Waksman R. Peroxisome proliferator-activated receptor gamma: its role in metabolic syndrome. Cardiovasc Radiat Med 2004; 5(2): 97-103.

[3] Day C. Thiazolidinediones: a new class of antidiabetic drugs. Mar Diabet Med 1999; 16(3):179-92.

[4] Hauner H. The mode of action of thiazolidinediones. Diabetes Metab Res Rev 2002;18 (Supp1 2): S10-S15.

[5] Dello Russo C, Gavrilyuk V, Weinberg G, et al. Peroxisome proliferator-activated receptor gamma thiazolidinedione agonists increase glucose metabolism in astrocytes. J Biol Chem 2003; 278(8): 5828-36.

[6] Preininger K, Stingl H, Englisch R, et al. Acute troglitazone action in isolated perfused rat liver. Br J Pharmacol 1999; 126(1): 372-8.

[7] Perez-Ortiz JM, Tranque P, Vaquero CF, et al. Glitazones differentially regulate primary astrocyte and glioma cell survival. Involvement of reactive oxygen species and peroxisome proliferator-activated receptor-gamma. Mar J Biol Chem 2004; 279(10): 8976-85.

[8] Spagnolo A, Grant EN, Glick R, Lichtor T, Feinstein DL. Differential effects of PPARgamma agonists on the metabolic properties of gliomas and astrocytes. Neurosci Lett 2007; 417(1): 72-7.

[9] Colca JR, McDonald WG, Waldon DJ, et al. Identification of a novel mitochondrial protein ("mitoNEET") cross-linked specifically by a thiazolidinedione photoprobe. Am J Physiol Endocrinol Metab 2004; 286(2): E252-E60.

[10] Dello Russo C, Polak PE, Mercado PR, et al. The heat-shock protein 90 inhibitor 17-allylamino-17-demethoxygeldanamycin suppresses glial inflammatory responses and ameliorates experi- mental autoimmune encephalomyelitis. J Neurochem 2006; 99(5): 1351-62.

[11] Phulwani NK, Feinstein DL, Gavrilyuk V, Akar C, Kielian T. 15-deoxy-Delta12,14-prostaglandin J2 (15d-PGJ2) and ciglitazone modulate Staphylococcus aureus-dependent astrocyte activation primarily through a PPAR-gamma-independent pathway. J Neurochem 2006; 99(5): 1389-402.

[12] Ciaraldi TP, Gilmore A, Olefsky JM, Goldberg M, Heidenreich KA. In vitro studies on the action of CS-045, a new antidiabetic agent. Metabolism 1990; 39(10): 1056-62.

[13] Ranganathan S, Kern PA. Thiazolidinediones inhibit lipoprotein lipase activity in adipocytes. J Biol Chem 1998; 273(40): 26117-122.

[14] Fulgencio JP, Kohl C, Girard J, Pegorier JP. Troglitazone inhibits fatty acid oxidation and esterification and gluconeogenesis in isolated hepatocytes from starved rats. Diabetes 1996; 45(11): 1556-62.

[15] Wang M, Wise SC, Leff T, Su TZ. Troglitazone: an antidiabetic agent, inhibits cholesterol biosynthesis through a mechanism independent of peroxisome proliferator-activated receptor-gamma. Diabetes 1999; 48(2): 254-60.

[16] Furnsinn C, Neschen S, Noe C, et al. Acute non-insulin-like stimulation of rat muscle glucose metabolism by troglitazone in vitro. $\mathrm{Br}$ J Pharmacol 1997; 122(7): 1367-74.

[17] Brunmair B, Gras F, Neschen S, et al. Direct thiazolidinedione action on isolated rat skeletal muscle fuel handling is independent of peroxisome proliferator-activated receptor-gamma-mediated changes in gene expression. Diabetes 2001; 50(10): 2309-15.

[18] Brunmair B, Staniek K, Gras F, et al. Thiazolidinediones, like metformin, inhibit respiratory complex I: a common mechanism contributing to their antidiabetic actions? Diabetes 2004; 53(4): 1052-9.

[19] Scatena R, Bottoni P, Vincenzoni F, et al. Bezafibrate induces a mitochondrial derangement in human cell lines: a PPAR-independent mechanism for a peroxisome proliferator. Chem Res Toxicol 2003; 16(11): 1440-7.

[20] Paddock ML, Wiley SE, Axelrod HL, et al. MitoNEET is a uniquely folded $2 \mathrm{Fe} 2 \mathrm{~S}$ outer mitochondrial membrane protein stabilized by pioglitazone. Proc Natl Acad Sci USA 2007; 104(36): 14342-7.

[21] Hou X, Liu R, Ross S, Smart EJ, Zhu H, Gong W. Crystallographic studies of human MitoNEET. J Biol Chem 2007; 282(46): 33242-6.

[22] Lin J, Zhou T, Ye K, Wang J. Crystal structure of human mitoNEET reveals distinct groups of iron sulfur proteins. Proc Natl Acad Sci USA 2007; 104(37): 14640-5.

[23] Wiley SE, Murphy AN, Ross SA, van der GP, Dixon JE. MitoNEET is an iron-containing outer mitochondrial membrane protein that regulates oxidative capacity. Proc Natl Acad Sci USA 2007; 104(13): 5318-23.

[24] Spagnolo A, Glick RP, Lin H, Cohen EP, Feinstein DL, Lichtor T. Prolonged survival of mice with established intracerebral glioma receiving combined treatment with peroxisome proliferator-activated receptor-gamma thiazolidinedione agonists and interleukin-2-secreting syngeneic/allogeneic fibroblasts. J Neurosurg 2007; 106(2): 299-305.

(C) Akar et al.; Licensee Bentham Open.

This is an open access article licensed under the terms of the Creative Commons Attribution Non-Commercial License (http://creativecommons.org/licenses/ by-nc/3.0/) which permits unrestricted, non-commercial use, distribution and reproduction in any medium, provided the work is properly cited. 